Estudios Románicos, Volumen 28, 2019, pp. 233-245

ISSN: 0210-491

eISSN: 1989-614X

DOI: https://doi.org/10.6018/ER/373971

\title{
EL SUPERESTRATO ROMÁNICO: LA HUELLA DEL ESPAÑOL, DEL FRANCÉS Y DEL OCCITANO EN EL SICILIANO CONTEMPORÁNEO
}

(Romance Superstratum: the trace of Spanish, French and Occitan in contemporary Sicilian)

\author{
José García Fernández \\ Universidad de Oviedo
}

\begin{abstract}
Sicily has always been a territory under dispute by multiple peoples that eventually managed to mold the culture of the inhabitants of the island. As a result, the Sicilian language absorbed the language of the ensuing settlers, particularly Spanish and French. In view of this, this article addresses the analysis of the Spanish, French and Occitan traces, three Romance variants that, through the semantic superstratum, have had an impact on the linguistic configuration of the Sicilian language. With the focus on the dialectal lexicon used by the Palermitan writer, Giuseppina Torregrossa, in her debut novel, L'Assaggiatrice, this study confirms what previous specialized authorities on the field observed in past times: that the Ibero-Romance and Gallo-Romance influence have been, and still are, key to the shaping of the Sicilian language, a linguistic variant that is most frequently used in insular literature regardless of the growing social interest in standard Italian after the unification.
\end{abstract}

Keywords: Romance linguistics; Italian dialectology; Literary plurilingualism; Semantic superstratum; Ibero-Romance influence; Gallo-Romance traces.

Resumen: Sicilia siempre ha sido un enclave disputado por múltiples pueblos que terminarían por modelar la cultura de sus habitantes. En consecuencia, el siciliano se embebió lingüísticamente de las hablas de los sucesivos pobladores de la isla, de entre los que caben destacar, entre otros, los españoles y los franceses. Atentos a esta realidad, este

* Dirección para correspondencia: José García Fernández, Departamento de Filología Clásica y Románica (Área de Filología Italiana) de la Facultad de Filosofía y Letras de la Universidad de Oviedo, Campus de Humanidades, calle Amparo Pedregal s/n, 33011, Oviedo, Principado de Asturias, España (garciafernandezjose@uniovi.es). 
artículo propone un acercamiento a los influjos del castellano, francés y occitano, tres variedades romances que, en forma de superestrato semántico, han influido en la configuración lingüística del siciliano. Sirviéndonos de las voces dialectales empleadas por la palermitana Giuseppina Torregrossa en su primera novela, L'Assaggiatrice, hemos podido corroborar cómo, al igual que hicieran antaño otros especialistas, tanto el dominio iberorrománico como el galorrománico siguen siendo determinantes en la conformación de la lengua siciliana, una variante lingüística aún empleada con frecuencia en la literatura isleña pese al creciente interés social por el italiano estándar desde la unificación del país.

Palabras clave: Lingüística románica; Dialectología italiana; Plurilingüismo literario; Superestrato semántico; Influjo iberorrománico; Influencia galorrománica.

\section{Introducción, objetivos y metodología}

Favorecida por su posición geoestratégica, Sicilia ha sido poblada a lo largo de su convulsa historia por numerosas comunidades sumamente divergentes. De estas raíces culturales emana su idiosincrasia, una manera de ser que demarca, afecta y determina a sus habitantes a la par que caracteriza la lengua que estos usan en su cotidianidad. No sorprende, por tanto, que el siciliano, la lengua autóctona de Sicilia ${ }^{1}$, presente una serie de particularidades diversas a las del resto de variedades lingüísticas itálicas (Ruffino 2013: 91-214). De acuerdo con esto, el propósito de este artículo es poner en valor el actual plurilingüismo literario siciliano y los estudios de dialectología italiana, un ámbito de especialización que ha pasado a ocupar un lugar secundario tras la unificación de Italia y la instauración del italiano estándar como lengua vehicular del estado. La oficialidad de esta koiné y el paulatino aprendizaje de la misma por parte de la sociedad italiana relegaron a un segundo plano las investigaciones dialectológicas, asociadas con frecuencia desde entonces al análisis de variantes lingüísticas rústicas que se han vinculado, por lo general, a contextos rayanos en la incultura (Marazzini 2018: 27-39).

De entre los notables influjos culturales que delimitan la "sicilianidad" lingüística, este artículo se centra concretamente en la influencia ejercida por el español, el francés y el occitano, tres romances que han modelado la lengua siciliana y que, al igual que el griego, el árabe y las lenguas germánicas (Ruffino 2013: 11-25, 35-47, 61), han condicionado la configuración lingüística de la isla. Es por ello que en el siguiente apartado se da cuenta de hasta qué punto estas lenguas románicas han dejado su huella en forma de superestrato léxico, utilizándose, a modo de corpus, la primera novela escrita por una de las más insignes y célebres autoras del panorama literario siciliano contemporáneo: L'Assaggiatrice (2010 [2007]), de Giuseppina Torregrossa. Esta elección responde a motivos puramente funcionales: al igual que han hecho otros escritores insulares como Andrea Camilleri, son muchas las isleñas que también han sabido volcar en sus escritos el plurilingüismo

1 Téngase presente que, de conformidad con los datos recogidos por Pellegrini en la Carta dei Dialetti d'Italia (1977), el siciliano quedaría agrupado dentro de los dialectos meridionales (específicamente, en el área meridional extrema), diferenciándose así del resto de variantes lingüísticas que conforman el dominio italorrománico: los dialectos septentrionales, el friulano, el toscano y el sardo. 
inherente a su zona de origen ${ }^{2}$. En consonancia con otras literatas sicilianas de la talla de Emma Dante, Torregrossa ha sabido conjugar la literatura, la lingüística y la cultura en su producción literaria, haciendo de su primera obra un valioso instrumento lingüístico en el que se recogen, a nivel diastrático y diatópico, los diversos registros idiomáticos de Sicilia (italiano estándar, italiano de Sicilia, siciliano) ${ }^{3}$. Por consiguiente, el examen atento de las voces empleadas por esta novelista en L'Assaggiatrice se revela útil, pues saca a la luz los rasgos definitorios de una variante lingüística, la siciliana, en la que incluso el adstrato del italiano estándar ha repercutido sobremanera en el modo de comunicarse de sus ciudadanos (D'Achile 2010 [2003]: 245-261; Mengaldo 2014 [1994]: 87-93).

Para alcanzar los objetivos propuestos, a nivel metodológico se ha hecho necesaria la utilización de diversos recursos electrónicos. La información recabada a través de internet ha sido extraída de las páginas de la enciclopedia italiana "Treccani", del diccionario del "Corriere della Sera" y de "Garzanti Linguistica". También se han utilizado la sección lingüística de la web "Dialettando", el vocabulario italiano-siciliano de "Terralab", el diccionario etimológico de términos meridionales presentes en autores italianos contemporáneos de la Universidad de Niza "Sophia Antipolis" (Francia) y el diccionario de la Real Academia Española (DRAE). La consulta de estas fuentes ha sido clave, puesto que están en constante actualización y recogen vocablos que no siempre aparecen incluidos en los diccionarios impresos. Además, estas herramientas electrónicas permiten colmar las dificultades derivadas de la escasez de materiales de corte dialectológico centrados en Sicilia, algunos de los cuales, como se verá a continuación, incluso se remontan al siglo XIX.

En consecuencia, ha sido igualmente perentorio recurrir al uso de diversos manuales en formato papel, gracias a los cuales se han podido completar y enriquecer los datos obtenidos en internet. En concreto, se han utilizado los siguientes volúmenes: Nuovo dizionario siciliano-italiano compilato da una società di persone di lettere (Mortillaro 1838), Dizionario siciliano-italiano (Rocca y Pasqualino 1839), Vocabolario numerico siciliano-italiano per l'interpretazione dei sogni ad uso dei giocatori del lotto (Pecorella 1985), Grammatica siciliana del dialetto e delle parlate (Pitrè y Wentrup 1996), Lingue e culture in Sicilia (Ruffino 2013) y Vocabolario Storico-Etimologico del Siciliano (VSES) (Varvaro 2014). No obstante, habida cuenta de la gran cantidad de información que habrá de manejarse en las subsecciones que engloban el próximo apartado, los ejemplos extraídos de la obra de Torregrossa se referenciarán señalando, entre paréntesis, el número de página al final de la cita. Cada voz se ilustrará -siempre y cuando los hubierecon un máximo de tres ejemplos; en caso de haber más, se indicará(n) en una nota al pie la(s) páginas(s) en la(s) que aparece(n). Las observaciones consignadas entre corchetes son de carácter personal y explicativo.

\footnotetext{
2 Esta característica es extrapolable a toda Italia. Piénsese, por ejemplo, en el uso de napolitanismos por parte de Elena Ferrante, una escritora contemporánea que muestra su propia identidad cultural a través del empleo de un dialecto ampliamente estudiado por Ledgeway (2009).

3 Se ha tenido en cuenta, por tanto, que "nonostante la superficie dell'Italia non sia vastissima, la variabile diatopica è [...] particolarmente importante: la ricchezza dei dialetti [...] ha avuto e continua ad avere riflessi notevoli sull'italiano che a quei dialetti si è sovrapposto, soprattutto sul piano fonetico e su quello lessicale, determinando la nascita degli italiani regionali" (D'Achile 2010 [2003]: 34).
} 


\section{El superestrato románico}

Como se ha venido anunciando, el influjo del dominio iberorrománico -sobre todo, del español-y del galorrománico ha moldeado el siciliano especialmente a nivel léxico. En virtud de ello, y sirviéndonos de los sicilianismos empleados por Torregrossa en L'Assaggiatrice, esta sección hace un recorrido teórico-práctico por el papel desempeñado por ambas áreas (ibérica, gala) en la actual configuración lingüística de Sicilia, un territorio en el que ilustres autoras como Giuseppina Torregrossa continúan empleando en sus escritos esta variante romance italorrománica como símbolo de identidad y sin ningún tipo de prejuicio. Conforme a este planteamiento, y a pesar de que hay un notable interés por fomentar y difundir el italiano estándar en la isla, es posible evidenciar cómo el siciliano no se utiliza ni residual ni minoritariamente. Más bien al contrario, su uso está teniendo cada vez una mayor fuerza en la cultura y en la literatura isleñas contemporáneas (Ruffino 2013: 866-880), un aspecto que da más entidad al siciliano como lengua vehicular de comunicación.

\subsection{El influjo iberorrománico: el caso del español}

La Corona de Aragón - cuya convergencia con la Corona de Castilla se inicia tras el matrimonio de los Reyes Católicos en 1469- tuvo un gran peso en el territorio insular tras los lamentables incidentes derivados de las Vísperas sicilianas (1282), un acontecimiento que condujo al asesinato de numerosos franceses en la isla y que, al mismo tiempo, causó el fin del reinado de Carlos de Anjou, siendo este sustituido por Pedro III de Aragón (Runciman 1986; Tramontana 1989; Natoli 2009). Ante esta coyuntura, fueron muchos los catalanes y los aragoneses que emigraron y optaron por instalarse en Sicilia, aspecto que, como cabe esperar, influyó considerablemente en la lengua cotidiana de sus habitantes. No obstante, si bien el influjo catalán se nota con claridad en el léxico siciliano (Grassi Privitera 1932; Varvaro 1974: 86-107), se percibe aún con mayor intensidad en el caso del español (Di Trapani 1963-1964; D’Agostino 1994: 791-824; Michel 1996; Núñez Méndez y Chakerian 2012: 249-273). La anexión del Reino de Sicilia a España tras la muerte de Fernando II de Aragón evidencia, de hecho, la relevancia que adquirió este último país, un estado que, sin embargo, nunca llegó a mostrar un excesivo aprecio hacia la isla.

La dejadez de la Corona española en la enseñanza y aprendizaje de la lengua y literatura estatales redujo, por tanto, la notoriedad del castellano en la isla, un idioma que, como apunta Ruffino (2013: 63-64), tan solo se utilizó en el ámbito cancilleresco -donde su uso no sería exclusivo, sino que conviviría con el latín y el italiano- y en actos comunicativos propios de la vida cotidiana (sobre todo entre políticos, militares, marineros, comerciantes, funcionarios, inquisidores, diplomáticos). Esta limitada esfera lingüística redujo, en ocasiones, el empleo del español a ambientes culturales de cierto renombre que poco tenían que ver con las circunstancias vitales de buena parte de los isleños. Aun así, precisamente por ser utilizado en registros más bien elevados, el castellano también contó en los siglos XVI-XVIII con una literatura propia en Sicilia, 
una literatura cuyos escritos -en su mayoría, traducciones- reflejaban, tal y como hace Torregrossa en L'Assaggiatrice, las interferencias lingüísticas que se producían entre las hablas locales y el español del momento.

Con arreglo a lo expuesto, no sorprende que el vocabulario español adoptado por los sicilianos esté compuesto mayoritariamente por voces que hacen referencia, bien a la esfera cotidiana, bien a una terminología de tipo armamentístico, económico, administrativo o inquisitorial ${ }^{4}$. De ahí que el superestrato castellano haya sido decisivo en Sicilia. La llegada de españoles a este territorio a partir del siglo XIII sirvió de base para la difusión de su lengua materna, una situación que propició una nueva coyuntura lingüística en la que el español se tornó esencial. La presencia de España no solo en Sicilia, sino también en toda la zona meridional de Italia, testimonió el poder y la autoridad que este país ejerció sobre el Mezzogiorno italiano, un dominio que a nivel lingüístico queda reflejado en algunos de los términos empleados por Torregrossa en L'Assaggiatrice ${ }^{5}$ :

- Aviri (Gana Di): Verbo siciliano en infinitivo (< lat. habere) acompañado de la construcción gana di (< esp. ganas de), it. 'avere voglia di', esp. 'tener ganas de'. Ejs.:

(1) La za Rosa oggi ha gana di [verbo conjugado en presente de indicativo] parlare (p. 13).

(2) Quello ha gana solo di [verbo conjugado en presente de indicativo] mangiare (p. 18).

(3) Avevo gana di [verbo conjugado en pretérito imperfecto de indicativo] fare quaccheccosa, tanto per non passare la giornata con una mappina nelle mani (p. 24) ${ }^{6}$.

- Livari (la GANA Di): Verbo siciliano en infinitivo (< lat. levare, procedente a su vez del término levis) acompañado de la construcción la gana di (< esp. las ganas de), it. 'togliere/levare la voglia di', esp. 'quitar las ganas de'. Ej.:

(1) A me questo freddo mi ha levato la gana di [verbo conjugado en passato prossimo] fottere (p. 137).

4 No obstante, si bien este repertorio léxico es predominante, el influjo español -palpable, por otro lado, en nombres relativos a oficios, a labores artesanales, a la caracterización del comportamiento humano o a la descripción de cualidades físicas y psíquicas- también se constató en otros aspectos: "Particolarmente interessante fu l'influsso della moda spagnola, più tra le denominazioni di vestimenti ufficiali dei magistrati e dei religiosi che tra $\mathrm{i}$ nomi dell'abbigliamento femminile [...] Il siciliano accolse anche molti iberismi legati alla cucina, oltre che numerosi esotismi di matrice coloniale. [...] [Inoltre, ci sono] voci relative ai più disparati campi della vita, dall'arredamento alla casa, agli attrezzi domestici, all'artigianato, alle feste, molte delle quali entrate sicuramente attraverso la vita di corte" (Ruffino 2013: 65).

5 En el texto se ha detectado un ejemplo en el que, si bien la influencia léxica es griega, la sufijación del término haya su origen en el español. Se trata del sustantivo fem. sg. timpulata < gr. $\tau u ́ \pi \tau \omega+$ esp. bofetada, it. 'schiaffo', esp. 'bofetón, guantazo'. Ej.: Lei allora lo doveva consolare per quelle timpulate che lui le stampava sulla faccia e sulle cosce (p. 30).

6 En los tres casos, al igual que en el resto de ejemplos con la construcción (la) gana di, el verbo está conjugado en italiano, mientras que el sintagma gana di aparece escrito en siciliano, una característica propia del italiano de Sicilia. Se pueden encontrar más ejemplos en las pp. 37, 49, 57, 65, 72, 111, 119. 
- Passari (la Gana di): Verbo siciliano en infinitivo (< lat. vulg. *passare, forma reconstruida por los romanistas y derivada del término lat. passus) acompañado de la construcción la gana di (< esp. las ganas de), it. 'passare la voglia di', esp. 'pasar las ganas de'. Ej.:

(1) Appena sentivo quell'odore di sugo fresco, il caldo del vapore che si alzava dal piatto, il ciauru della ricotta salata che si squagliava sopra ai maccheroni, mi passava la gana di [verbo conjugado en pretérito imperfecto de indicativo] mangiare (p. 68).

- Simana: Sustantivo fem. sg. < esp. semana, it. 'settimana'. Ej.:

(1) Anciluzza si deve sposare, non ti vediamo da una simana (p. 11).

- VINIRI (GANA DI): Verbo siciliano en infinitivo (< lat. venire) acompañado de la construcción gana di (< esp. ganas de), it. 'far venire la voglia di', esp. 'venir las ganas de, apetecer'. Ej.:

(1) A me dà del lei, mi chiama signora, anche se qui tutti mi dicono Anciluzza; più mi chiama signora e più mi viene gana di [verbo conjugado en presente de indicativo] entrarci in confidenza (p. 93).

- Vossìa: Pronombre personal, resultado de la contracción de las formas sicilianas vossignurìa, vostra signuria < esp. vusía, vuestra señoría', it. 'Lei', esp. 'usted'. Ej.: (1) Signuri' m'avìti a pirdunare, vossia è la figghia di... (p. 86).

- Zita: Sustantivo fem. sg. < esp. cita (aunque con un notable cambio de significado $\left.{ }^{8}\right)$, it. 'ragazza, fidanzata', esp. 'chica, novia'. Ej.:

(1) Zà Rosa, un pochino di vino che porta bene alla zita (p. 13).

\subsection{El influjo galorrománico: francés y occitano}

En tanto que centro cultural hegemónico de Europa y Occidente a lo largo de la historia, Francia ha contribuido de manera destacada al enriquecimiento léxico del siciliano (Zolli 1977: 55-62; Dardi 1986: 21-36; Morgana 1994: 671-720; Valenti 2004, 2005: 63-146, 2011). Sin embargo, a diferencia de otros influjos lingüísticos, no fueron los galos quienes introdujeron de forma directa algunas de las voces propias de su vocabulario, sino los soldados y colonos del norte de Italia, trabajadores que acudieron a la isla por petición de los normandos asentados en Sicilia desde el siglo XI. En consecuencia, tal y como advierte Ruffino (2013: 48), la paulatina inclusión de rasgos galorromá-

7 Como señala Varvaro (2014: 1179), vossìa es un préstamo o calco del español vusía, contracción de vuestra señoría. De hecho, este filólogo añade que existen formas análogas en numerosos dialectos italianos, remitiendo incluso a la voz italiana vossignoria (actualmente en desuso).

8 La utilización del sustantivo zita precedido del verbo fari acarrea igualmente un pequeño cambio semántico: fari zita significa 'casarse', en it. 'sposarsi'. Las palabras de Vito, personaje tartamudo de L'Assaggiatrice, dan cuenta de este matiz léxico en la siguiente oración interrogativa: ej. AAAAnn Anncicici lululluzza mmm mi ppppp piaci, tttt'ha fffafari zizizita cucucu mmmia? (p. 33), esp. 'A A A Ann Anncicici lululluzza mmm me ggggg gustas, ¿ttttee quiquieres cacacasar conconmigo?' 
nicos en Sicilia (debilitamiento de las sílabas átonas, sonorización de las consonantes oclusivas intervocálicas, lenición celta, frecuente caída de consonantes finales, habitual presencia de los fonemas vocálicos /y, ø, æ/) se produjo gracias al contacto del siciliano con variedades lingüísticas romances pertenecientes al área septentrional italiana, una zona que, aun no compartiendo todas las características propias del habla francesa, sí que contaba con considerables similitudes gramaticales.

Y si bien la emigración al sur de la península itálica (ss. XII-XIII) favoreció una integración gradual de voces galas -sobre todo, de términos relacionados con la vida cotidiana o con el ámbito agrícola, ganadero y alimenticio-, la relevancia que Francia adquirió en el contexto internacional entre los siglos XVII y XIX fue fundamental a la hora de asimilar nuevos préstamos, esta vez ya no procedentes del francés antiguo, sino del moderno (Ruffino 2013: 49, 67-70).

De todas maneras, si se quiere establecer un análisis detallado del dominio galorrománico, es necesario resaltar que esta área lingüística no se limita al francés. El occitano ("erróneamente" denominado "provenzal" hasta hace poco"), aun no siendo oficial en Francia, sigue representando a día de hoy uno de los principales símbolos de identidad de la zona centro-meridional del país ${ }^{10}$. Su bagaje histórico da cuenta de su trascendencia. Con el occitano, las regiones del Mediodía francés asistieron en la Edad Media a un florecimiento cultural determinado por el cada vez más fulgurante empleo de este romance. El occitano se convertiría en la lengua basilar de una nueva corriente literaria fuertemente determinada por el concepto del amor cortés, o, lo que es lo mismo, en una lengua presente en numerosas composiciones literarias cuya temática hizo de la poesía trovadoresca un movimiento de renombre. En relación con la influencia occitana sobre el siciliano, cabe señalar:

Nel siciliano, $[\ldots]$ i provenzalismi entrarono per vie diverse: alcuni, facendo già parte del francese antico, furono portati dai Normanni, altri furono introdotti in Sicilia dagli Angioini, altri ancora dipesero dalle scelte lessicali dei poeti della Magna

$9 \quad$ Nótese que hasta el siglo XIX no puede tacharse de error la denominación comúnmente utilizada para referirse a esta lengua, pues no existía un término "neutro" con el que aludir al occitano tal y como se concibe a día de hoy. Es más, los catalanes del siglo XIII llegaron a llamar limosín al occitano, habida cuenta del prestigio que los trovadores procedentes de este espacio dialectal alcanzaron durante este periodo. No sorprende, pues, que "se ha[ya] confundido por autores de la mejor nota el lenguaje lemosín, el provenzal y el catalán, creyéndolos uno mismo" (AA. VV. 1831: 738). En cualquier caso, ateniéndonos a los parámetros lingüísticos actuales, cabe matizar que el provenzal es un dialecto del occitano, lengua que, en verdad, cuenta con las siguientes variantes dialectales: provenzal (como ya se ha señalado), alpino-delfinés, aquitano-gascón y auvergnat-limosín. En relación con este aspecto, los estudios de P. Bec (1963, 1970: 395-462, 1973) y la Gramática histórica provenzal de J. Ramón Fernández González (1985) ofrecen una amplia panorámica de este dominio lingüístico (dialectos, subdialectos, isoglosas, etc.).

10 La lengua francesa, de hecho, penetró con enorme lentitud en esta zona, debido, entre otras causas, a la permanente inestabilidad y a los conflictos territoriales incesantes. Fue la ordenanza de Villers-Cotterêts (1539) la que consiguió devaluar de forma indirecta las distintas variedades del occitano aún habladas entonces. A este respecto, además de los múltiples estudios lingüísticos sobre el francés llevados a cabo por estudiosos de la talla de Brunel y de Brunot, destaca la contribución de Klinkenberg (1999: 228), quien señala cómo la penetración del francés en el sur occitanófono era ya una realidad en el siglo XIII; en concreto, desde la cruzada albigense hasta finales del siglo XVI casi todos los dominios occitanos pasaron a formar parte del Reino de Francia. Por este motivo, la lengua occitana cayó rápidamente en desuso a partir del siglo XV, una situación que también cambió su estatus sociolingüístico. 
Curia di Federico II, emuli - attraverso l'impiego del siciliano al posto del provenzale - dei modelli provenzali (ma in quest'ultimo caso, si trattò per lo più di usi letterari circoscritti) (Ruffino 2013: 58).

Este uso del occitano como referente literario y de cultura ha motivado la división en dos bloques de los términos de origen galo usados por Torregrossa en L'Assaggiatrice. Por ello, se ha optado por recoger en primer lugar las voces procedentes del francés -ya sea antiguo o moderno- para luego proceder a presentar aquellas derivadas del occitano. Los ejemplos extraídos del texto se presentan justo a continuación.

\subsubsection{Voces de origen francés}

- Baglio: Sustantivo masc. sg. adaptado fonéticamente al italiano, derivado del término siciliano bagliu/bagghiu $<$ fr. antiguo bail $(e)^{11}$, it. 'edificio che contiene la corte o il cortile'12. Ejs.:

(1) Nella piazza principale, l'unica, un abbeveratoio grande per le bestie, un bar, due piccoli edifici adibiti a pensione, i cancelli di un orto meraviglioso, sempre chiuso, l'ufficio postale, il tabaccaio e l'arco del baglio Isonzo, che permette di entrare in un piazzale pieno di fichi, eucalipti e oleandri. Dietro al baglio si sviluppa un agglomerato di case (p. 41).

(2) Il forno a legna, un piccolo negozio di alimentari e uno di ceramiche, che in Sicilia non mancano mai, si affacciano sul cortile del baglio (p. 42).

(3) C'è dipinto un vecchio baglio con una palma e un muretto a secco, sembra un'oasi nel deserto (p. 76) ${ }^{13}$.

- Catamiàrisi: Verbo siciliano en infinitivo < fr. démener (o incluso del término siciliano arriminari, procedente a su vez del fr. remuer), it. 'muovere, smuovere', esp. 'mover(se), sacudir'. Ej.:

(1) Capisco che devo farmi forza, catamiàrmi e iniziare un nuovo ciclo della mia vita (p. 33).

- 'Ntamata: Adjetivo fem. sg. derivado del verbo siciliano 'ntamari < fr. antiguo entamer, it. 'sbalordita', esp. 'asombrada, abrumada'. Ej.:

(1) I giorni successivi alla sparizione di Gaetano li passo come in un sogno, un poco imbambolata, 'ntamata (p. 29).

11 Si bien bail(e) es un préstamo del francés antiguo que, de forma genérica, tenía un sentido militar, Varvaro (2014: 104) precisa que fue en Normandía donde esta voz terminó por asumir la significación propia del it. 'cortile' (esp. 'patio').

12 Intraducible en castellano, este término cuenta con un equivalente en árabe andalusí: hisn/husun (cfr. Sénac 2012). En particular, esta voz hace alusión a una construcción siciliana fortificada en la que vivían los campesinos y sus patrones en las épocas de cosecha y recolección. De ahí que esta palabra esté ligada al ámbito agrícola y feudal, y dé cuenta de cuán importante era el latifundio en Sicilia.

13 Se pueden encontrar más ejemplos en las pp. 87, 104. 
- PARrino: Sustantivo masc. sg. adaptado fonéticamente al italiano, derivado del término siciliano parrinu < préstamo de época normanda derivado del fr. antiguo parrin, procedente a su vez del lat. patrinu $(m)$, it. 'prete', esp. 'párroco'14. Ejs.:

(1) Non mi sono mai pentita; e poi pure il parrino mi ha assolta (pp. 13-14).

(2) Si tratta piuttosto di una specie di vendetta trasversale messa in atto contro al parrino e ai suoi noiosissimi insegnamenti (pp. 42-43).

(3) Don Antonio, il parrino, che nei primi tempi mi guardava in cagnesco, si è ammorbidito (p. 49) ${ }^{15}$.

- VAnedda: Sustantivo fem. sg. < préstamo de época normanda derivado del fr. antiguo venelle, it. 'vicolo, piccola via', esp. 'vereda'. Ej.:

(1) Nell'aria si sente solo l'odore del mare e della cacca delle mucche che pascolano a piano della vanedda (p. 125).

\subsubsection{Voces de origen occitano}

- FARFAnTARİa: Sustantivo fem. sg. derivado del término siciliano farfanti $<$ occ. antiguo forfar (o incluso del fr. forfaire), it. 'menzogna', esp. 'mentira, engaño, canallada'. Ej.: (1) È morto soffocato dalla parola buttana che gli è rimasta ferma nel cannarozzo. Non mi sono mai pentita; e poi pure il parrino mi ha assolta, in fondo è l'unica farfantaria della mia vita (pp. 13-14).

- 'Ntrusciato: Adjetivo masc. sg. adaptado fonéticamente al italiano y derivado del término siciliano 'ntrusciatu, procedente a su vez del verbo siciliano 'ntrusciari $<$ occ. antiguo trossar (o incluso del fr. antiguo trousser o de la voz siciliana truscia), it. 'infagottato, avolto, attillato', esp. ‘envuelto, ceñido, apretado'. Ej.:

(1) [La Romana] ancheggiando in un vestito azzurro, intrusciato attorno ai fianchi, si avvicina alla porta del mio negozio (p. 93).

- Picciotto: Sust. masc. sg. adaptado fonéticamente al italiano, derivado del término siciliano picciottu < occ. pichot ${ }^{16}$, it. 'giovanotto, ragazzo', esp. 'joven, chaval, chico'. Ejs.:

(1) Nulla a che vedere con quel giovane uomo dagli occhi di lava, il sorriso di un vulcano e il portamento di un picciotto scapestrato (p. 98).

(2) All'improvviso quel picciotto scanzonato ed estraneo prese le sembianze di un principe del deserto (p. 98).

14 Nótese, en cualquier caso, que ni la voz del fr. antiguo parrin ni la del fr. moderno parrain han tenido nunca el significado de 'sacerdote', sino el de 'padrino'. Por tanto, tal y como declara Varvaro (2014: 745), aun conservándose el vocablo parrino en Sicilia y en Calabria, el significado del término habría experimentado una evolución semántica ignota al francés, un cambio léxico propio de la Italia meridional que acabaría derivando en su significación actual: 'padre spirituale, confessore'.

15 Aparece otro ejemplo en la p. 57.

16 De todas formas, se considera igualmente que esta voz deriva del lexema *pikk-, una raíz "alla quale risalgono diverse voci rom., tra cui it. piccolo", y a la que se le añadió el sufijo -ottu (Varvaro 2014: 754, 756-757). 
No obstante, también es frecuente el empleo de la forma apocopada picciò (it., esp. 'tesoro') como apelativo cariñoso. Ej.:

(1) Picciò, quaccheccosa mi avevano detto, ma che ne potevo sapere che la prima notte avrei goduto così (p. 13).

\section{Conclusiones}

Los sicilianismos utilizados por Torregrossa en L'Assaggiatrice contribuyen a ahondar en la esencia ibérica y gala de una isla que se ha visto condicionada lingüísticamente por el superestrato léxico de ambos dominios. La fuerte presencia en el sur de Italia de la Corona de Aragón primero, y de la Corona de Castilla después, se tradujo en la inclusión de palabras catalanas, y esencialmente castellanas, desde el siglo XIII en adelante. Por desgracia, España nunca llegó a mostrar un excesivo aprecio por Sicilia, una desatención que derivó en una deficiente difusión de la lengua y literatura estatales y que redujo, por tanto, el ámbito de uso del español a las cancillerías -en convivencia con el latín y el italiano-y a actos comunicativos propios de la vida común. Así pues, no resulta extraño que el repertorio léxico español adoptado por los sicilianos se componga en su mayoría de términos relativos a estados de ánimo pasajeros propios del día a día (aviri/viniri gana di, livarilpassari la gana di). A estos vocablos se suman igualmente otros nombres concernientes a la caracterización de la condición humana (señálese, a título ilustrativo, la voz zita, relativa al estado civil de las mujeres) o al desarrollo y paso del tiempo (simana). Y si bien la mayor parte de estos conceptos se emplea en situaciones de comunicación habituales, se ha podido detectar, asimismo, cómo en ciertas ocasiones las voces castellanas han dado origen a formas de cortesía propias de registros formales. Basta pensar en el pronombre personal vossia para percatarse de ello.

Francia, por su parte, en tanto que centro cultural de prestigio durante siglos, también contribuyó al enriquecimiento léxico del siciliano. Sin embargo, a diferencia de los pueblos ibéricos, su influjo no fue directo. En realidad, los verdaderos responsables resultaron ser los soldados y los colonos del norte de Italia, quienes, acudiendo a Sicilia por petición de los normandos (ss. XI-XII), difundieron, en consonancia con los españoles, el empleo de voces esencialmente relativas al ámbito cotidiano. Esta singularidad queda reflejada en la utilización de términos vinculados al mundo de la construcción y de las comunicaciones (baglio, vanedda), de adjetivos que describen sentimientos y estados de ánimo ('ntamata) o de palabras que aluden a usuales y respetados cargos eclesiásticos (parrino). Y aunque, en el fondo, estos vocablos no compartían todas las características propias del habla francesa, no pueden considerarse ajenos a este dominio, pues, al fin y al cabo, contaban -como todavía hoy sucede en el área septentrional italiana-con significativas semejanzas gramaticales.

Por último, símbolo de identidad de la zona centro-meridional de Francia, el occitano también fue clave en el devenir del siciliano. El florecimiento cultural que experimentó el Mediodía francés durante la Edad Media favoreció el empleo de un romance que sentó las bases de una corriente literaria determinada por el amor cortés. El éxito 
alcanzado por este movimiento literario fomentó la divulgación del occitano en múltiples enclaves románicos, alcanzando incluso la distante y singular Sicilia. Su notoriedad fue tal que, al igual que en los casos precedentes, múltiples coloquialismos y voces occitanas pasaron a utilizarse en la isla para retratar la rutina de la vida isleña. En este artículo se ha podido apreciar esta particularidad gracias al examen de los términos farfantarìa, 'ntrusciato y picciottolpicciò, tres vocablos de uso común y metafórico en la comunicación cotidiana que, en sintonía con las palabras castellanas y francesas, reflejan hasta qué punto el occitano, junto al castellano y al francés, sigue siendo determinante en la actual configuración lingüística de Sicilia.

\section{BIBLIOGRAFÍA}

AA.VV. (1831): Diccionario geográfico universal (t. I). Barcelona: Imprenta de José Torner. BEC, Pierre (1963): La langue occitane. Paris: Presses Universitaires de France.

(1970): Manuel pratique de philologie romane (t. I). Paris: Éditions A. et J. Picard. (1973): Manuel pratique d'occitan moderne. Paris: Éditions A. et J. Picard.

CORRIERE DELLA SERA (n. d.): Dizionario italiano (edizione online tratta da: il Sabatini Coletti). [http://dizionari.corriere.it/dizionario_italiano/; 07/05/2019].

D'ACHILE, Paolo (2010 [2003]): L'italiano contemporaneo. Bologna: Il Mulino.

D’AGOSTINO, Alfonso (1994): "L'apporto spagnolo, portoghese e catalano". Storia della lingua italiana. Torino: Einaudi, vol. 3, 791-824.

DARDI, Andrea (1986): "Elementi francesi moderni nei dialetti italiani". Elementi stranieri nei dialetti italiani. Atti del XIV Convegno del Centro di Studio per la Dialettologia Italiana. Pisa: Pacini, vol. 1, 21-36.

DI TRAPANI, Maria (1963-1964): Gli ispanismi del siciliano (tesi di laurea). Palermo: Università degli Studi di Palermo.

DIALETTANDO (n. d.): Dizionario dei dialetti d'Italia. [http://www.dialettando.com/ dizionario/dizionario.lasso; 07/05/2019].

FERNÁNDEZ GONZÁLEZ, José Ramón (1985): Gramática histórica provenzal (prólogo de Álvaro Galmés de Fuentes). Oviedo: Servicio de Publicaciones de la Universidad de Oviedo.

GARZANTI LINGUISTICA (n. d.): Dizionario italiano. [https://www.garzantilinguistica.it/ricerca/; 07/05/2019].

GRASSI PRIVITERA, Giovan Battista (1932): Somiglianze della lingua catalano-castigliana col dialetto siciliano. Palermo: Scuola Tip. Boccone Del Povero.

ISTITUTO DELL'ENCICLOPEDIA ITALIANA FONDATA DA GIOVANNI TRECCANI (n. d.): Vocabolario. [http://www.treccani.it/vocabolario/; 07/05/2019].

KLINKENBERG, Jean-Marie (1999): Des langues romanes (préface par Willy Bal). Bruxelles: Éditions De Boeck Université.

LEDGEWAY, Adam (2009): Grammatica diacronica del napoletano. Tübingen: Max Niemeyer Verlag. 
MARAZZINI, Claudio (2018): L'italiano è meraviglioso. Come e perché dobbiamo salvare la nostra lingua. Milano: Rizzoli.

MENGALDO, Pier Vincenzo (2014 [1994]): Storia dell'italiano del Novecento. Bologna: Il Mulino.

MICHEL, Andreas (1996): Vocabolario critico degli ispanismi siciliani. Palermo: Centro di studi filologici e linguistici siciliani.

MILANESI, Luigi; MOROLDO, Arnaldo (2010): Méridionalismes chez les auteurs italiens contemporains. Dictionnaire étimologique (avec la collaboration du Zu Mimi; Webmaster Claude Hennebois). [http://sites.unice.fr/site/henneboi/ SVG-lirces/langues/real/dialectes/index.htm; 07/05/2019].

MORGANA, Silvia (1994): "L'influsso francese". Storia della lingua italiana. Torino: Einaudi, vol. 3, 671-720.

MORTILLARO, Vincenzo (a cura di) (1838): Nuovo dizionario siciliano-italiano compilato da una società di persone di lettere (vol. I). Palermo: Tipografia del Giornale Letterario.

NATOLI, Luigi (2009): I Vespri siciliani. Palermo: Flaccovio Editore.

NÚÑEZ MÉNDEZ, Eva; CHAKERIAN, Raven (2012): "Estudio lingüístico-comparativo del siciliano y el español”. Literatura y Lingüística. Núm. 25: 249-273.

PECORELLA, Giusto (1985): Vocabolario numerico siciliano-italiano per l'interpretazione dei sogni ad uso dei giocatori del lotto (integrato e aggiornato da Salvatore Ciancio). Roma: Edizioni Mediterranee.

PELLEGRINI, Giovan Battista (1977): Carta dei Dialetti d'Italia. Pisa: Pacini.

PITRÈ, Giuseppe; WENTRUP, Christian F. (1996): Grammatica siciliana del dialetto e delle parlate (a cura di Giovanni Ruffino). Palermo: Flaccovio Editore.

REAL ACADEMIA ESPAÑOLA (2014): Diccionario de la lengua española (23. ${ }^{\mathrm{a}} \mathrm{ed}$.). [http://dle.rae.es; 07/05/2019].

ROCCA, Rosario; PASQUALINO, Francesco (1839): Dizionario siciliano-italiano. Catania: Pietro Giuntini.

RUFFINO, Giovanni (a cura di) (2013): Lingue e culture in Sicilia (vol. I). Palermo: Centro di studi filologici e linguistici siciliani, Regione Siciliana - Assessorato Regionale dei Beni Culturali e dell'Identità Siciliana.

RUNCIMAN, Steven (1986): I Vespri siciliani. Storia del mondo mediterraneo alla fine del tredicesimo secolo (traduzione di Pasquale Portoghese). Bari: Edizioni Dedalo.

SÉNAC, Philippe (ed.) (2012): Histoire et archéologie de l'occident musulman (VII ${ }^{e}-X V^{e}$ siècle): Al-Andalus, Maghreb, Sicile. Toulouse: Méridiennes.

TERRALAB (n. d.): Vocabolario italiano-siciliano. [http://www.terralab.it/sishilianu/ Vocabolario.htm; 07/05/2019].

TORREGROSSA, Giuseppina (2010 [2007]): L'Assaggiatrice. Soveria Mannelli (Catanzaro): Rubbettino.

TRAMONTANA, Salvatore (1989): Gli anni del Vespro. L'immaginario, la cronaca, la storia. Bari: Edizioni Dedalo. 
VALENTI, Iride Marianita Bernadette (2004): Vocabolario storico-etimologico dei gallicismi e dei francesismi del siciliano (tesi di laurea). Catania: Università degli Studi di Catania.

(2005): "Francesismi nel lessico culinario del siciliano". Bollettino dell' Atlante Linguistico Italiano. Núm. 29, III serie: 63-146.

(2011): Gallicismi nella cultura alimentare della Sicilia. Palermo: Centro di studi filologici e linguistici siciliani.

VARVARO, Alberto (1974): "Prima ricognizione dei catalanismi nel dialetto siciliano". Medioevo Romanzo. Núm. 1: 86-107.

(2014): Vocabolario Storico-Etimologico del Siciliano (VSES) (vol. 2). Strasbourg: Éditions de linguistique et de philologie, Centro di studi filologici e linguistici siciliani.

ZOLLI, Paolo (1977): "I francesismi dei secoli XVIII e XIX nei dialetti italiani". Interferenza linguistica. Atti del Convegno della Società Italiana di Glottologia. Pisa: Giardini Editori e Stampatori, 55-62.

\section{PERFIL ACADÉMICO-PROFESIONAL}

José García Fernández es licenciado en Filología Románica por la Universidad de Oviedo, institución en la que también ha cursado sus estudios de Máster Universitario Internacional en Lengua Española y Lingüística y en la que ha desarrollado una tesis doctoral dentro del Programa de Doctorado en Investigaciones Humanísticas (especialidad en Filología Italiana). Actualmente está adscrito al Área de Filología Italiana del Departamento de Filología Clásica y Románica de la Universidad de Oviedo, donde continúa su labor docente e investigadora gracias a la obtención de una ayuda "Severo Ochoa" para la formación en investigación y docencia del Principado de Asturias (convocatoria 2016, referencia: PA-17-PF-BP16053). Asimismo, cuenta en su haber con diversas publicaciones relacionadas con sus áreas de interés. Su campo de estudio se centra en la lingüística y la gramática italianas, en la traducción italiano-español y en la literatura y cultura sicilianas. Forma parte del proyecto de I+D "Ausencias II. Escritoras italianas inéditas en la Querella de las mujeres (siglos XV al XX)".

Fecha de recepción: 30/04/2019

Fecha de aceptación: 15/07/2019 